\title{
Long-Lasting Current Transient Phenomena in TlBr
}

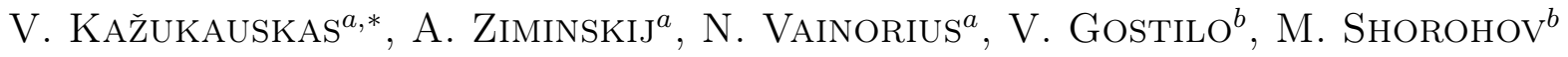 \\ AND V. BOZHKO ${ }^{c}$ \\ ${ }^{a}$ Semiconductor Physics Department and Institute of Applied Research \\ Vilnius University, Saulètekio al. 9/3, LT-10222, Vilnius, Lithuania \\ ${ }^{b}$ Brucker Baltic, Riga, Latvia \\ ${ }^{c}$ Volyn National University, Lutsk, Ukraine
}

\begin{abstract}
The persistent conductivity effects in TlBr were evidenced at the temperatures below about $200 \mathrm{~K}$ after the intrinsic light excitation. They were associated with the filling of the trapping states having thermal activation energy of about $0.08-0.12 \mathrm{eV}$ and accompanied by the appearance of the pronounced maximum of the thermally stimulated currents. In this state the enhanced photoconductivity was observed that could be thermally quenched above about $180 \mathrm{~K}$. The quenching is initiated by the emptying of the trapping states having activation energy of $0.63-0.65 \mathrm{eV}$.
\end{abstract}

PACS: 71.55.-i, 72.20.Jv, 72.40.+w, 72.60.+g, 72.80.Jc

\section{Introduction}

Thallium bromide $(\mathrm{TlBr})$ is a promising material for $\mathrm{X}$ - and $\gamma$-ray spectroscopy because of its wide bandgap $(2.68 \mathrm{eV})$, high density $\left(7.56 \mathrm{~g} / \mathrm{cm}^{3}\right)$ and high atomic numbers (Tl: 81 and Br: 35) [1,2]. Such properties ensure a high photon stopping efficiency. Nevertheless one of the problems hindering practical applications of $\mathrm{TlBr}$ is presence of the ionic conductivity, which sensitively depends on the temperature [3] and makes device characteristic unstable in time. Apart from that carrier transport and defect properties of $\mathrm{TlBr}$ are still relatively little investigated depending on the excitation conditions. As below about $240 \mathrm{~K}$ ionic conductivity of $\mathrm{TlBr}$ becomes negligible [4], such situation is useful for the analysis of electronic transport. Therefore we had investigated photoconductivity of $\mathrm{TlBr}$ at the temperatures close to liquid nitrogen temperature.

\section{Samples and experiment}

TIBr single crystals were grown by the BridgmanStockbarger method. Spectral dependences of photoconductivity, thermally stimulated currents and current transients were investigated in the temperature range from $100 \mathrm{~K}$ to $300 \mathrm{~K}$. The photoconductivity spectra were analysed using the Lucovsky photoionization model $[4,5]$. Carrier transport and trapping were investigated by the thermally stimulated current (TSC) spectroscopy as described in $[6,7]$. To evaluate possible effects caused by several closely located trapping centres, the multiple

* corresponding author; e-mail: vaidotas.kazukauskas@ff.vu.lt heating technique was used, that enables sequential emptying of the levels, thus enhancing possibilities of their discrimination [6].

\section{Results and discussion}

At $300 \mathrm{~K}$ photoconductivity in $\mathrm{TlBr}$ is low, therefore any defect-related features could not be identified by photospectroscopy [8]. By decrease of the dark current with temperature, the effect of photoconductivity increases, enabling identification of defect levels, depending on the sample prehistory, i.e., its excitation by light and/or voltage [4]. In Fig. 1 spectral dependences of photoconductivity are presented at different temperatures: $240 \mathrm{~K}$ and $115 \mathrm{~K}$. The spectra were scanned first by increasing quantum energy, afterwards by decreasing it. It can be seen that by increasing the quantum energy, spectra measured at both temperatures coincide in detail, though the dark current is lower at lower temperature.

A maximum related with the band-to-band generation appears at about $2.75 \mathrm{eV}$. Current drop at the high-energy side is associated with the increasing absorption and intensifying surface recombination. By fitting the experimental curves using the Lucovsky photoionization model [5] the following photoionization energies were identified: $0.55,0.83,1.1,1.32$, and $1.65 \mathrm{eV}$. The recharge of the trap with the activation energy of $1.65 \mathrm{eV}$ could induce carrier mobility variations [4]. Characteristically, defect bands in the region between $1 \mathrm{eV}$ and $2.5 \mathrm{eV}$ were dependent on sample prehistory. The defect-related shoulders at about 0.83 and $1.1 \mathrm{eV}$ used to appear if the spectra were scanned from the high to the low quantum energies. Meanwhile influence of the levels at 1.32 and $1.65 \mathrm{eV}$ used to decrease if the sample was kept bi- 


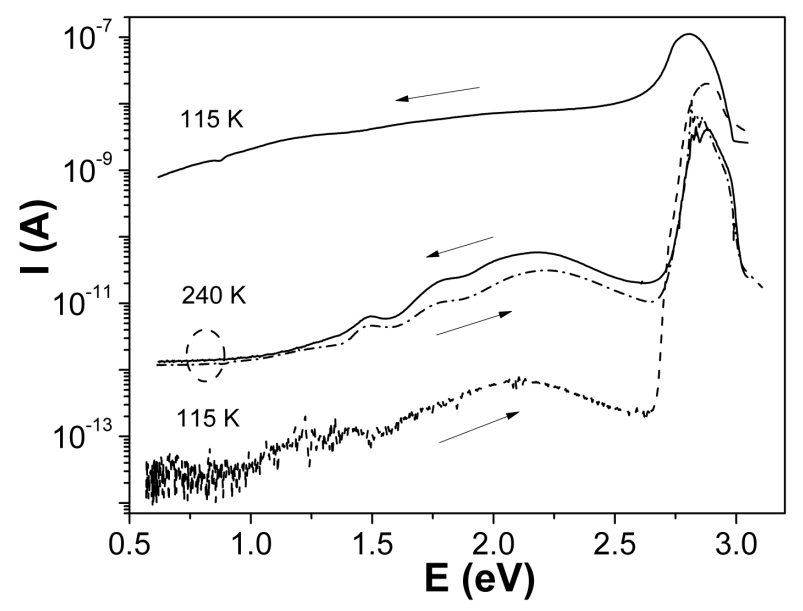

Fig. 1. Spectral dependences of photoconductivity of the same sample at different temperatures. First the spectra were scanned by increasing quantum energy, next by decreasing it as it is indicated by the arrows.

ased for several hours. Similar complicated defect structure was also revealed by photoluminescence (PL) in [9]. The luminescence in the range $1.5-2.0 \mathrm{eV}$ was observed under X-ray excitation and was ascribed to the diffusion-controlled recombination in [9], meanwhile PL at $1.1 \mathrm{eV}$ was observed in the short-lived absorption spectra under pulsed electron beam excitation in [10] and it was explained to be due to the hole trapped at cation vacancy $\left(\mathrm{Tl}^{2+} \mathrm{Vc}^{-}\right)$. This centre can be created if hole is trapped by a pre-irradiation vacancy, as well as result from the radiation-induced Frenkel pair formation $\left(\mathrm{Tl}^{2+} \mathrm{Vc}^{-}\right.$and interstitial thallium atom $\mathrm{Tl}_{\mathrm{i}}^{0}$ ). Our results indicate that such vacancy can also be light-induced, even though the intensity of light, passing through the monochromator, is low. The more detailed analysis of the results at $240 \mathrm{~K}$ is presented in [4].

In contrast, at the temperatures below $180 \mathrm{~K}$, after the excitation of the sample by the intrinsic light, its photosensitivity remains by about four orders of magnitude higher than before the excitation. Moreover, no defect "fingerprints" are seen. This is caused by the appearance of the slow photocurrent relaxation called persistent photoconductivity (PPC) [11]. The relaxation of the photoconductivity could be approximated by a sum of three exponents with the time constants ranging from about $100 \mathrm{~s}$ up to $2500 \mathrm{~s}$. Characteristically, current did not decrease to zero, but was saturating at the constant level. The PPC phenomenon is related with the inability of the light generated carriers to recombine, because they become trapped in trapping states that might be related with potential inhomogeneities of the band edges. Therefore carriers become separated in space, and prior to recombination they have to gain energy to escape from the traps and to move in space [11]. However, in [11] there is stated that the relaxation of the current usually follows the single exponential dependence. In contrast, in our case a sum of three exponents was necessary to fit the experimental dependence, indicating the quite complex effect. Even the single stretched exponent was not enough for the fitting.

To analyse these slow trapping states the thermally stimulated currents were investigated. In Fig. 2 experimental TSC is indicated by the dashed line. Appearance of two current peaks with different activation energies is seen at lower temperatures. Close to the room temperature TSC curves is coinciding with the dark current having activation energy of about $0.65 \mathrm{eV}$. To reveal details of the peaks the multiple heating was used as presented in Fig. 3. At lower temperatures a wide and high TSC peak appears, having thermal activation energy values of about $0.065-0.08 \mathrm{eV}$. In the following heating cycles the increase of these values up to $0.11-0.12 \mathrm{eV}$ occurs. Such increase could be explained by the fact that, upon thermal generation of carriers from the potential wells of the band gap, the effective depth of the emptied wells is increasing in each temperature scan. After these shallow trapping states are emptied, carrier generation from deeper traps with the thermal activation energy of about $0.33 \mathrm{eV}$ begins. The emptying of this level occurs at about $220 \mathrm{~K}$, as it is implied by the flattening of the TSC curve. After that the thermal generation with the activation energy of $0.65 \mathrm{eV}$ starts which is close to the values of $0.68-0.7 \mathrm{eV}$ reported in $\mathrm{TIBr}[8,12]$. We have used these experimental values as model parameters to fit the TSC curve as described, e.g., in [13]. The effects of the single processes are presented in Fig. 2 by the dotted lines, meanwhile the resulting curve is shown by the solid line.

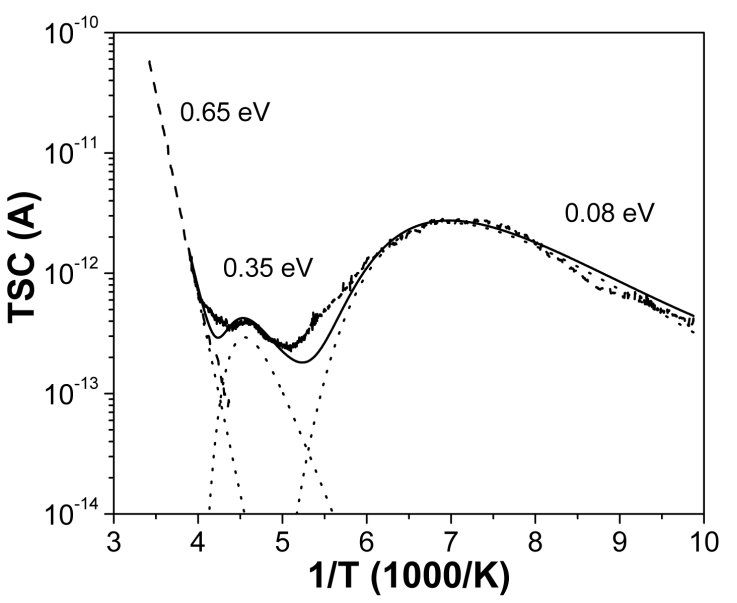

Fig. 2. Thermally stimulated current spectra (dashed line) and its fitting (solid line) representing a sum of three thermally stimulated processes (dotted lines) with different activation energy values.

Presence of the defect states, the role of which in carrier recombination depends on their filling, is demonstrated by appearance of the thermal quenching phenomenon of the photocurrent. In Fig. 4 temperature 


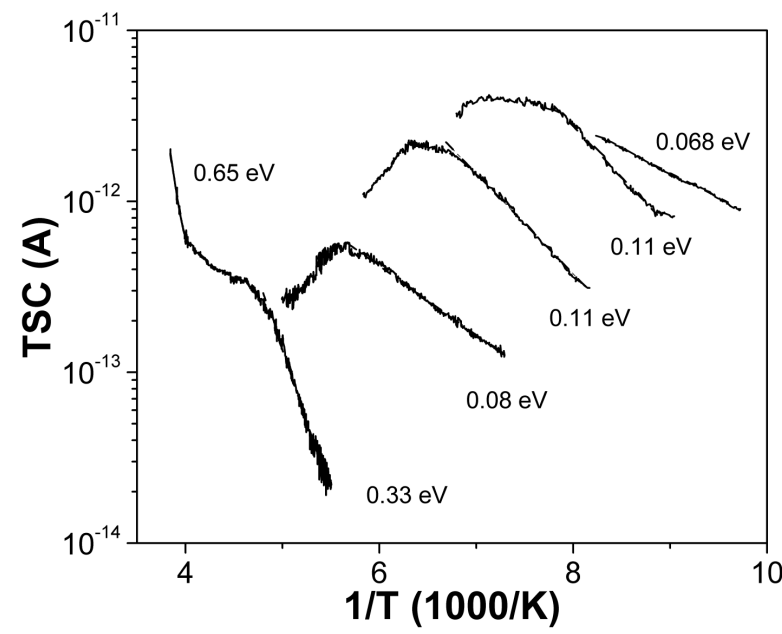

Fig. 3. Thermally stimulated current spectra (solid lines) measured by multiple heating. Numbers in figure indicate effective thermal excitation energy values.

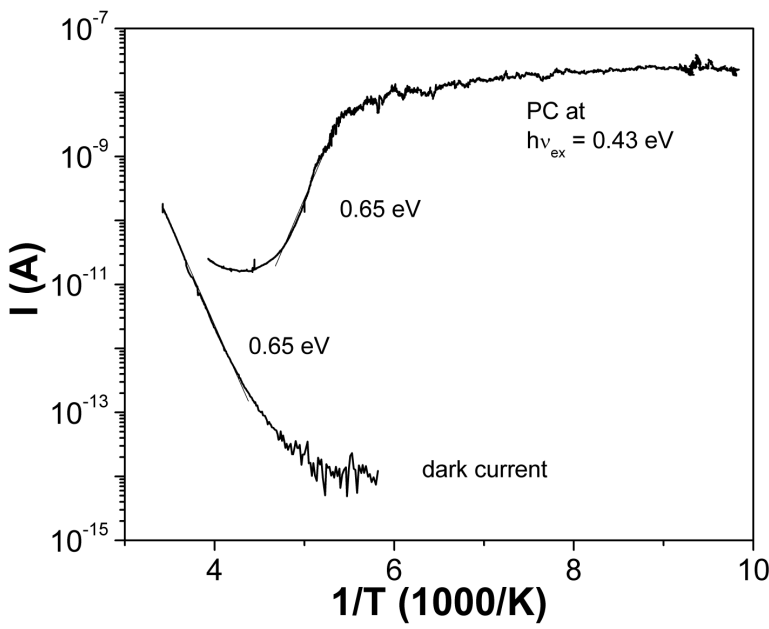

Fig. 4. Temperature dependences of the dark current and photocurrent upon band-to-band light excitation scanned from low to high temperatures.

dependences of the dark current and photocurrent upon band-to-band light excitation are demonstrated. They were scanned from low to high temperatures. The photocurrent is much higher than the TSCs and remains nearly constant up to about $180 \mathrm{~K}$. Above that point it starts to decrease. The thermal quenching of photoconductivity is well known, in particular in $\mathrm{A}_{2} \mathrm{~B}_{6}$ semicon- ductors [14]. It is caused by the thermal emptying of the recombination centres, which, when filled, cannot recombine the carriers. Characteristically, activation energy values of both thermal quenching and the dark current coincide within errors, indicating that thermal quenching might be initiated by emptying of the trap at about $0.65 \mathrm{eV}$.

\section{Acknowledgments}

This work was supported by the Lithuanian and Ukrainian Ministries of Education and the Research Council of Lithuania (project No. TAP-21, TAP-63 and MIP-11321). A. Ziminskij and N. Vainorius acknowledge Student Research Fellowship Award from the Lithuanian Science Council.

\section{References}

[1] P.J. Sellin, Nucl. Instrum. Methods Phys. Res. A 513, 332 (2003).

[2] K. Hitomi, M. Matsumoto, O. Muroi, T. Shoji, Y. Hirarate, J. Cryst. Growth 225, 129 (2001).

[3] T. Onodera, K. Hitomi, T. Shoji, Nucl. Instrum. Methods Phys. Res. A 568, 433 (2006).

[4] V. Kažukauskas, A. Ziminskij, J.V. Vaitkus, V. Gostilo, M. Shorohov, Nucl. Instrum. Methods Phys. Res. A 607, 123 (2009).

[5] G. Lucovsky, Solid State Commun. 3, 299 (1965).

[6] G. Kavaliauskienè, V. Kažukauskas, V. Rinkevičius, J. Storasta, J.V. Vaitkus, R. Bates, V. O'Shea, K.M. Smith, Appl. Phys. A 69, 415 (1999).

[7] J.G. Simmons, G.W. Taylor, Phys. Rev. B 5, 1619 (1972).

[8] V. Kažukauskas, A. Jurgilaitis, J.-V. Vaitkus, Proc. SPIE 6596, C5960 (2007).

[9] L. Grigorjeva, D. Millers, Nucl. Instrum. Methods Phys. Res. B 191, 131 (2002).

[10] L. Grigorjeva, D. Millers, Electrochem. Soc. Proc. 25, 438 (1998).

[11] M.K. Sheinkman, A.Y. Shik, Sov. Phys.-Semicond. 10, 128 (1976).

[12] J. Vaitkus, J. Banys, V. Gostilo, S. Zatoloka, A. Mekys, J. Storasta, A. Zindulis, Nucl. Instrum. Methods Phys. Res. A 546, 188 (2005).

[13] V. Kažukauskas, Semicond. Sci. Technol. 19, 1373 (2004).

[14] R.H. Bube, Photoconductivity of Solids, Wiley, New York 1960 